\title{
openheart Catheter ablation for the management of atrial fibrillation: current technical perspectives
}

\author{
Kathryn Lauren Hong (D) ,1,2 Jazlyn Borges, ${ }^{2}$ Benedict Glover ${ }^{1,2}$
}

To cite: Hong KL, Borges J, Glover B. Catheter ablation for the management of atrial fibrillation: current technical perspectives. Open Heart 2020;7:e001207. doi:10.1136/ openhrt-2019-001207

Received 13 November 2019 Revised 16 March 2020 Accepted 6 April 2020

\section{Check for updates}

(C) Author(s) (or their employer(s)) 2020. Re-use permitted under CC BY-NC. No commercial re-use. See rights and permissions. Published by BMJ.

${ }^{1}$ Cardiology, University of Toronto, Toronto, Canada ${ }^{2}$ Cardiology, Sunnybrook Health Sciences Centre, Toronto, Canada

\section{Correspondence to} Dr Benedict Glover; ben.glover@ hotmail.com

\begin{abstract}
Atrial fibrillation (AF) is the most common arrhythmia encountered in clinical practice, with a prevalence that increases alongside the ageing population worldwide. The management of $A F$ involves restoration of sinus rhythm through antiarrhythmic drug therapy. Yet, these medications have only modest efficacy in achieving longterm success, have not shown to result in a mortality benefit, are frequently not tolerated and have associated adverse side effects. Therefore, catheter ablation has become a valuable treatment approach for $\mathrm{AF}$ and even a viable first-line strategy in select cases. Traditionally, the combination of radiofrequency energy and a threedimensional electroanatomical mapping system has been used to guide catheter ablation for AF. However, singleprocedural efficacy and long-term outcomes still remain suboptimal for many patients, particularly those with persistent or long-standing AF. Recent advances in ablation technology and strategy, therefore, provide new procedural approaches for catheter-based treatment with the aim of overcoming current challenges in procedural duration and overall success. The aim of this paper was to provide an updated review of the current practices and techniques relating to ablation for $A F$ and to compare the use of these strategies for paroxysmal and persistent AF.
\end{abstract}

\section{INTRODUCTION}

Identifying the optimal strategy for the treatment of atrial fibrillation (AF) continues to be an evolving challenge for clinicians as the initiation and maintenance of $\mathrm{AF}$ occur as a result of complex interactions between arrhythmia triggers, changes in the atrial substrate and alterations in the autonomic nervous system activity. ${ }^{1}$ For patients undergoing catheter ablation, the optimal set of ablation lesions depend primarily on the distribution of these factors and the clinical type of $\mathrm{AF}^{2}$

Pulmonary vein isolation (PVI) is the recommended treatment for drug refractory symptomatic $\mathrm{AF}$ or in patients who are intolerant or unwilling to take antiarrhythmic drug therapy. ${ }^{3}$ However, the success of catheter ablation is limited and depends largely on patient characteristics. ${ }^{4}$ Indeed, while
PVI can be achieved in a significant proportion of patients with paroxysmal AF with a $60 \%-80 \%$ clinical success rate, ${ }^{5}$ results remain suboptimal in patients with persistent and long-standing persistent $\mathrm{AF}$ who are often refractory to catheter ablation or require multiple repeat procedures. In patients with paroxysmal AF, ablation successfully targets triggers that are predominantly located in the pulmonary veins (PVs) and less commonly non-PV triggers. Accordingly, circumferential PVI, with confirmation of both entrance and exit block, remains the cornerstone of this procedure.

Conversely, in patients with persistent AF, the atrial substrate is complicated by atrial wavelets, multiple macro re-entry circuits, and localised sources, ${ }^{67}$ leading to suboptimal procedural outcomes. ${ }^{8}$ The most effective ablation strategy in patients with persistent and long-standing persistent AF is currently unknown despite the fact that several techniques have been attempted with minimal or no success. A summary of triggers responsible for the initiation and propagation of $\mathrm{AF}$ is displayed in figure 1.

In this review, we will provide an overview of evolving techniques for the treatment of catheter ablation, while focusing on the more recent advances.

\section{Catheter technology and energy sources}

To date, a variety of energy modalities have been employed for the catheter ablation of AF including radiofrequency (RF), cryoablation (CB), microwave, high-intensity focused ultrasound and electroporation. Notably, the two most commonly used sources in clinical practice are RF and CB.

$\mathrm{RF}$ energy is generated at a frequency of $500-1000 \mathrm{KHz}$ and transmitted between the catheter tip and an electrode patch positioned on the skin. The maximum amount of resistive energy occurs within approximately $2 \mathrm{~mm}$ of the catheter tip and is either transmitted to the endocardial surface of the heart 


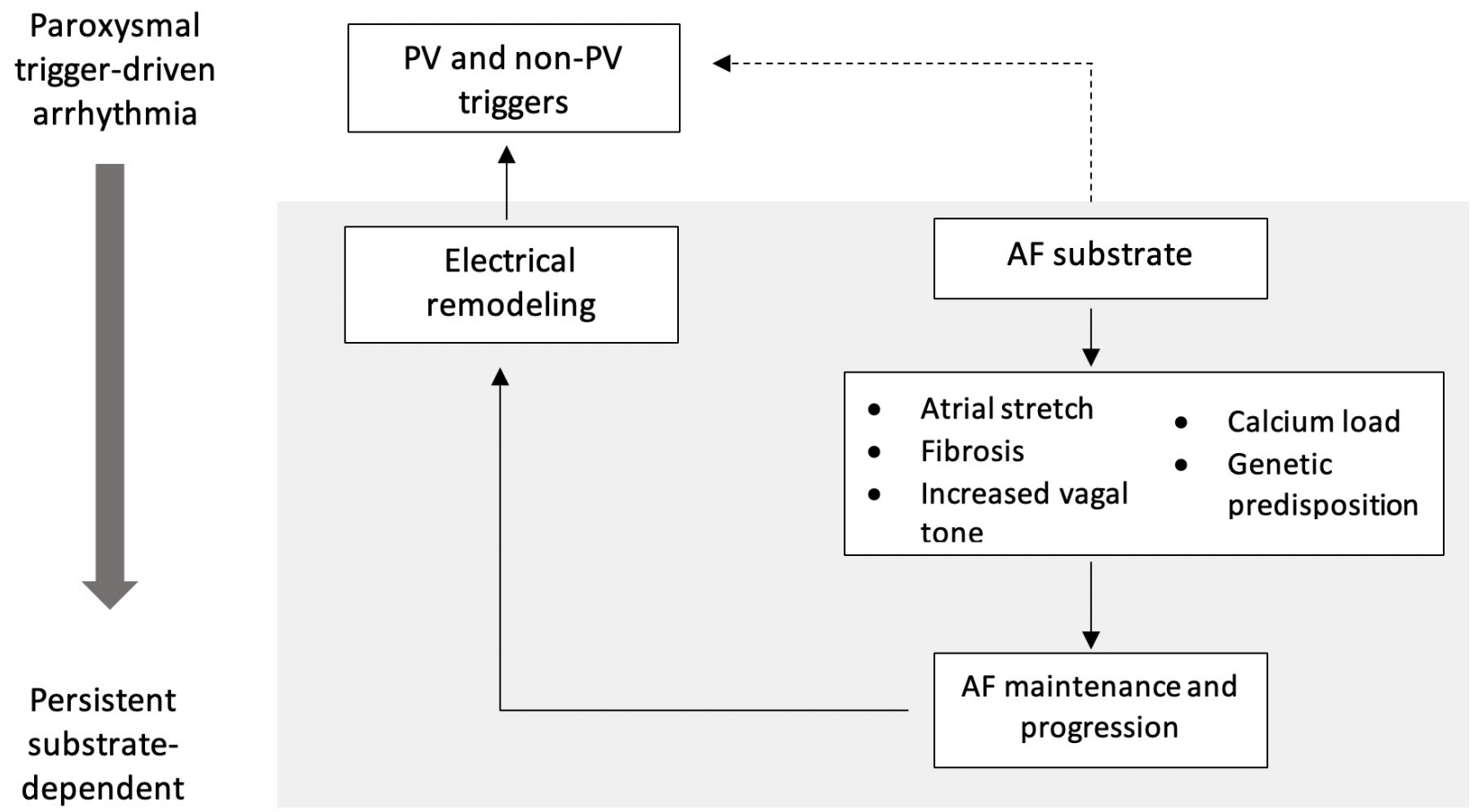

Figure 1 Distribution of triggers responsible for the initiation of AF. AF, atrial fibrillation; PV, pulmonary vein.

or the blood. The majority of lesion formation occurs as a result of conductive heat, which is inversely proportional to the distance from the tip of the electrode. CB on the other hand involves three phases of tissue damage. The first occurs during delivery of $\mathrm{CB}$ and is known as the freezing-thawing phase. As the temperature drops below $-15^{\circ} \mathrm{C}$, microscopic extracellular ice formation occurs, followed by intracellular ice formation when the temperature drops below $-40^{\circ} \mathrm{C}$ and results in localised tissue damage. As thawing occurs, there is fusion of the ice crystals with mircothrombi and platelet aggregation. Subsequently, the hemorrhagic-inflammatory phase occurs with localised tissue inflammation and oedema and finally, the replacement-fibrosis phase takes place and a fibrotic scar develops.

When compared with RF energy, patients randomised to either $\mathrm{CB}$ or RF groups had similar success rates and safety outcomes at 1.5 years follow-up. The results of the large multicentre Cryobaloon or Radiofrequency Ablation for Paroxysmal Atrial Fibrillation (FIRE AND ICE) trial, ${ }^{9}$ therefore, validate the use of $\mathrm{CB}$ as an alternative to conventional RF ablation for the treatment of paroxysmal AF. While the results of this trial may have been limited by the combined use of both first-generation noncontact force and second-generation contact force (CF) catheters, freedom from AF was still observed in $64.1 \%$ of subjects undergoing RF ablation and $65.4 \%$ of those undergoing CB. Additional 30 months freedom from AF data supported the non-inferiority of $\mathrm{CB}$ to RF ablation. It is important to note that cryoballoon ablation has been associated with significantly higher radiation doses due to the need for PV angiography to confirm complete PV occlusion and ensure optimal tissue freezing. ${ }^{10}$ Nonetheless, more than 35000 CB-based ablations have been performed worldwide to date. ${ }^{11}$

More recently high-power short-duration (HPSD) RF has been studied to assess if this is more effective and safer than conventional power settings. It has been postulated that HPSD lesions may minimise the impact of catheter stability as the lesion is delivered over a shorter period of time which may increase the consistency of the lesions with less localised oedema. By using the method there is more immediate localised tissue heating and with less conductive heat which may result in collateral damage. Ablation at $90 \mathrm{~W}$ delivered over $4 \mathrm{~s}$ per lesion has been shown to be effective (reduced linear gaps and non-transmural injury as well as increased lesion to lesion consistency) and safe in a preclinical model and superior to $20 \mathrm{~W}$ delivered over $20 \mathrm{~s}$ in the atrium. ${ }^{12}$

HPSD lesion diameters have been shown to be significantly larger and deeper when compared with standard settings in an in silico simulation study. ${ }^{13}$

\section{Ablation catheters}

Current catheter technology can be divided into a single tip irrigated catheter (generally $3.5-4.0 \mathrm{~mm}$ ) which may or may not use CF or a single-shot device which generally has multiple electrodes and is positioned within the PV antra in order to deliver energy over a region of tissue. 
Lesion creation depends on catheter-to-tissue contact, the quality of which is conventionally assessed via impedance drop during RF application. However, these parameters may not provide a full depiction of actual electrode-tissue contact ${ }^{14}$ and creating continuous effective circumferential lesions remains a clinical challenge. More recently, the adoption of CF-sensing catheters enables rapid and accurate confirmation of the completeness of PV antrum isolation, leading to improved outcomes and fewer repeat procedures. ${ }^{15-17}$ This is achieved through direct assessment of cathetermyocardial contact and directionality during the delivery of RF ablation. Catheter-tissue CF can be measured at the catheter tip either with fibreoptic or magnetic sensors which are able to measure the force between the distal catheter electrode and the tissue or a localised impedance calculation algorithm which does not provide CF but indicates wall contact versus no wall contact. The local impedance drop has a more accurate correlation with lesion size than global impedance drop. ${ }^{18}$

During a procedure, certain CF parameters can be measured in real time. This includes amplitude and orientation of real-time CF (recorded every $100 \mathrm{~ms}$ ), average force (Fav in gram-force, g), force time integral index measured as the product of real-time force and ablation time and lesion index, a proprietary index combining CF, RF application duration and RF current.

The effectiveness of a CF-sensing catheter has previously been investigated in ex vivo models and in vivo experimental studies. While these results were promising, there is no consensus that the use of CF catheters imparts better efficacy for PVI in clinical applications, particularly with respect to reducing the rate of complications. Nonetheless, real-time CF-sensing RF catheter ablation utilising CF of $10-20 \mathrm{~g}$ has been previously correlated with significantly reduced rates of AF recurrence in paroxysmal AF when compared with PVI without CF monitoring. ${ }^{19}$ Likewise, in patients with persistent AF, CF-guided ablation has been related to significantly reduced $\mathrm{AF}$ recurrence rates during 1-year follow-up as compared with non-CF-guided ablation, $27.6 \%$ vs $46.4 \%$, respectively. ${ }^{20}$ To date, countless studies still fail to demonstrate clear differences in ablation outcomes or procedural safety. ${ }^{21} 22$ First-pass PVI, however, does appear to be more likely with CF technology and occurrences of acute PV reconnection may also be diminished. $^{2324}$ This is because continuous catheter-tissue CF can be measured, which not only ensures accurate placement of the catheter at the onset of the procedure, but also enables further detection of catheter dislodging or shifting in real time. Future studies should aim to establish the optimal RF power and lesion duration required of CF-sensing catheters to ensure adequate lesion formation, while avoiding collateral tissue injury.

Although several single-shot devices have been used in the past with differing effects and complications, there are a number of single-shot devices currently being developed which are yet to be released on the clinical market.

\section{TECHNIQUES FOR THE MANAGEMENT OF PAROXYSMAL AF} Isolation of PV triggers

In patients with paroxysmal $\mathrm{AF}$, catheter ablation is concentrated on eliminating AF triggers, which originate within the PVs in $80 \%-94 \%$ of patients with $\mathrm{AF}^{25}$ Evaluation of PV electrical activity and identification of residual conduction gaps are performed using a circular multipolar catheter with a focus on PV antral electrical activity. Although the use of these catheters has previously been shown to result in lower rates of AF recurrence when compared with an RF catheter alone, ${ }^{26}$ the orientation of the PV to the left atrium (LA) is complex and may result in additional RF energy delivery beyond that which is required. A novel mapping catheter which allows for bipole recording both along and across the splines (HD Grid Mapping Catheter Sensor Enabled, Abbott Technologies, Mineapolis, USA), has been shown to enhance the accuracy of substrate mapping by providing simultaneous assessments of conduction voltage, activation and directionality. High-resolution mapping using the HD Grid has recently demonstrated efficacy in the assessment of bidirectional block and signal differentiation in first-time and redo PVI. ${ }^{27}$ However, while acute electrical disconnection between the PV and LA is an established endpoint and may indicate isolation after lesion delivery, there are cases where PV connection is concealed by local oedema. ${ }^{28}$ Three-dimensional (3D) mapping systems are widely utilised to support the procedure and can confirm the completeness of PVI by creating a detailed voltage map. In general, wide antral circumferential ablation is performed in order to achieve PVI.

\section{Non-PV trigger elimination}

The identification and elimination of non-PV triggers are important, particularly in repeat procedures, where PVI continues to be present. The majority of these non-PV triggers are located in the superior vena cava (SVC), coronary sinus and crista terminalis or at the posterior LA wall as well as the left atrial appendage (LAA) ${ }^{29}$ Specifically, isolation of the SVC has been evaluated as an adjunct to PVI. In one study of 320 consecutive patients with either paroxysmal or persistent AF, 90\% of those with paroxysmal AF undergoing SVC isolation were free of atrial tachycardia (AT) compared with $77 \%$ of those without isolation. ${ }^{30}$ However, these results are yet to be replicated in other randomised populations.

In patients without spontaneously firing non-PV triggers, a high-dose of isoproterenol (ie, infusion of $20-30 \mu \mathrm{g} / \mathrm{min}$ or bolus infusion of $10-20 \mu \mathrm{g}$ ) is commonly used to provoke them. Cardioversion of AF during lowdose isoproterenol infusion is also used in many cases. However, the protocol for provoking and subsequently localising the non-PV triggers is yet to be standardised. For patients with paroxysmal AF, there is little evidence to support the benefit of routine ablation beyond PVI in the absence of documented extrapulmonary triggers or coexisting atrial tachyarrhythmias in patients undergoing an index ablation. 


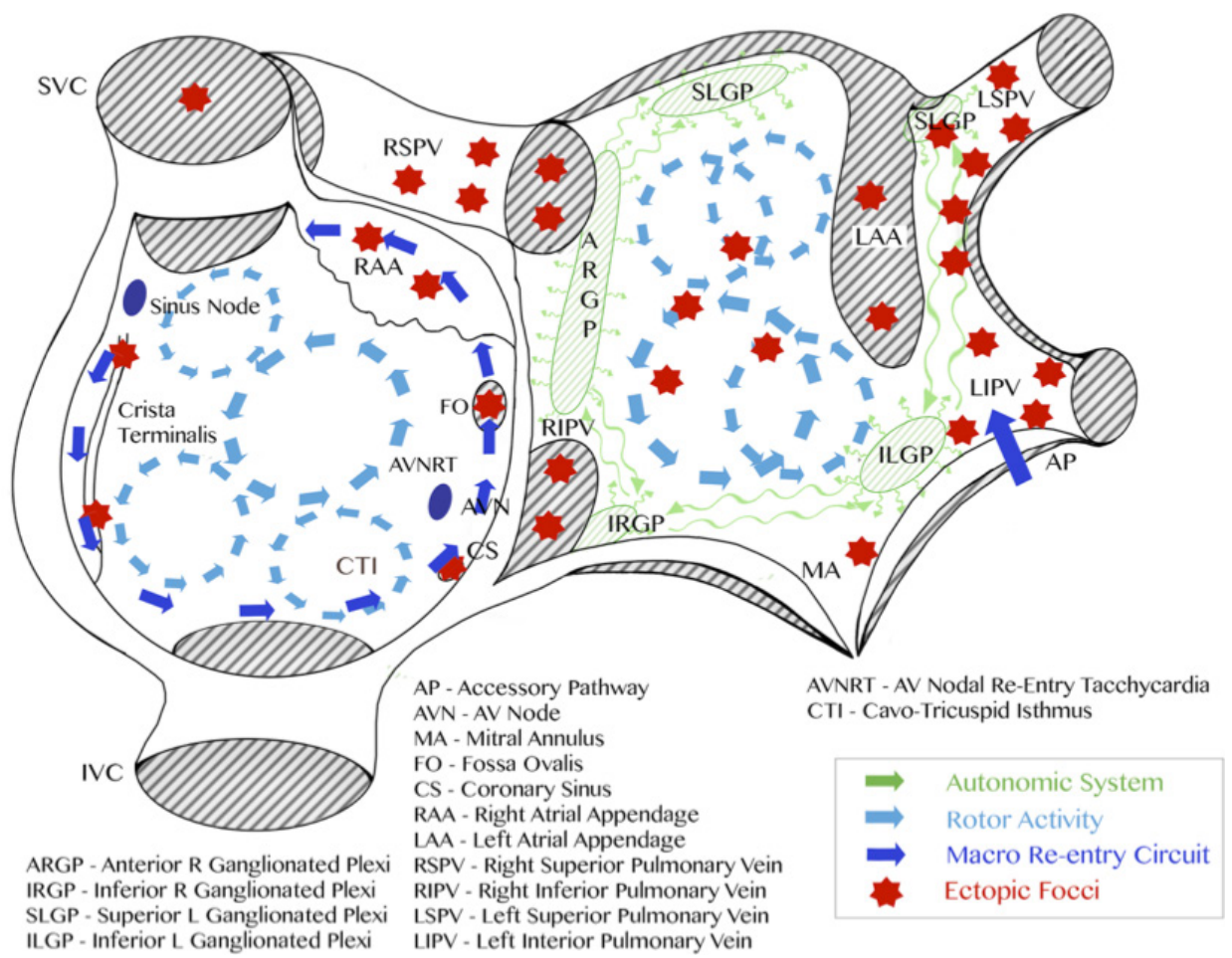

Figure 2 Potential mechanisms in the pathophysiology of persistent AF. AF, atrial fibrillation.

\section{MANAGEMENT OF PERSISTENT AF}

The effectiveness of PVI in patients with persistent and long-standing persistent $\mathrm{AF}$ has been reported as low as $21 \%{ }^{31}$ The basis for these results is that the sites of $\mathrm{AF}$ initiation and maintenance may be diffuse in all four PVs and their antrum and conduction recurrence in only one $\mathrm{PV}$ can lead to AF recurrence. ${ }^{32}$ The pathogenesis of persistent $\mathrm{AF}$ is complex and cannot be characterised by the same markers of paroxysmal AF (anisotropic conduction, autonomic innervation of the heart, embryogenesis of thoracic veins and interspersion of inhomogeneous tissue). The purported mechanisms of persistent $\mathrm{AF}$ are summarised in figure 2.

The main techniques which have been studied for the management of persistent $\mathrm{AF}$ include the elimination of complex fractionated atrial electrograms (CFAEs), linear lesions, rotor ablation and scar homogenisation. This has raised questions of whether trigger-based ablation strategies through PVI should be performed alone or in combination with substrate-based strategies, such as CFAEs. Yet, as recently reported, the use of additional ablation lesion sets such as CFAEs and linear lesions fail to improve ablation success.

\section{CFAE ablation}

Defragmentation of the LA is based on the elimination of all multicomponent electrograms of short cycle length, such as CFAEs. CFAEs are most often defined as continuous atrial activity, complex fractionated potentials or lowvoltage electrograms with a short cycle length of $<120 \mathrm{~ms}$ over a $10 \mathrm{~s}$ period. Therefore, their ablation usually leads to prolongation of AF cycle length, resulting in conversion into AT or sinus rhythm. ${ }^{33}$ However, the endpoints of CFAE ablation are not clearly defined, resulting in lowintraprocedural reproducibility of CFAE elimination and limited clinical success rates. ${ }^{34}$

Previous studies have demonstrated that CFAE originate along the regions of slow conduction, functional block and pivot points. ${ }^{35}$ Non-PV ectopies have also been found in similar locations as the CFAEs, with $25 \%$ of CFAEs in the LA and $57 \%$ of CFAEs in the right atrium related to non-PV triggers after PVI. ${ }^{36}$ These abnormal conduction patterns then serve as potential targets for ablation. Data suggest that CFAE ablation beyond PVI may not necessarily enhance single procedure efficacy in ablating persistent or long-standing persistent AF. In the RASTA Study, the addition of LA CFAE ablation with PVI was less efficacious to both PVI plus ablation of non-PV triggers using a standard approach as well as the standard protocol plus empirial ablation of common non-PV AF trigger sites (fossa ovalis, SVC and mitral annulus). ${ }^{37}$

Despite multiple investigations, the optimal treatment strategy for persistent AF remains unknown and the addition of substrate-based ablation strategies has failed to demonstrate conclusive benefit in subsequent trials. Results of the CHASE-AF Study ${ }^{38}$ and the recently published Alster-Lost-AF Study have demonstrated no clinical benefit of a combined approach of PVI plus additional substrate modification through CFAE ablation and linear ablation over stand-alone PVI. ${ }^{39}$ Of 118 patients with persistent or long-standing persistent $\mathrm{AF}$ who were assigned PVI alone or combined PVI and substrate modification, there was no statistically significant difference observed in recurrence rates ( $46 \%$ and $43 \%$, respectively). The multicentre, randomised STAR-AF II trial produced 

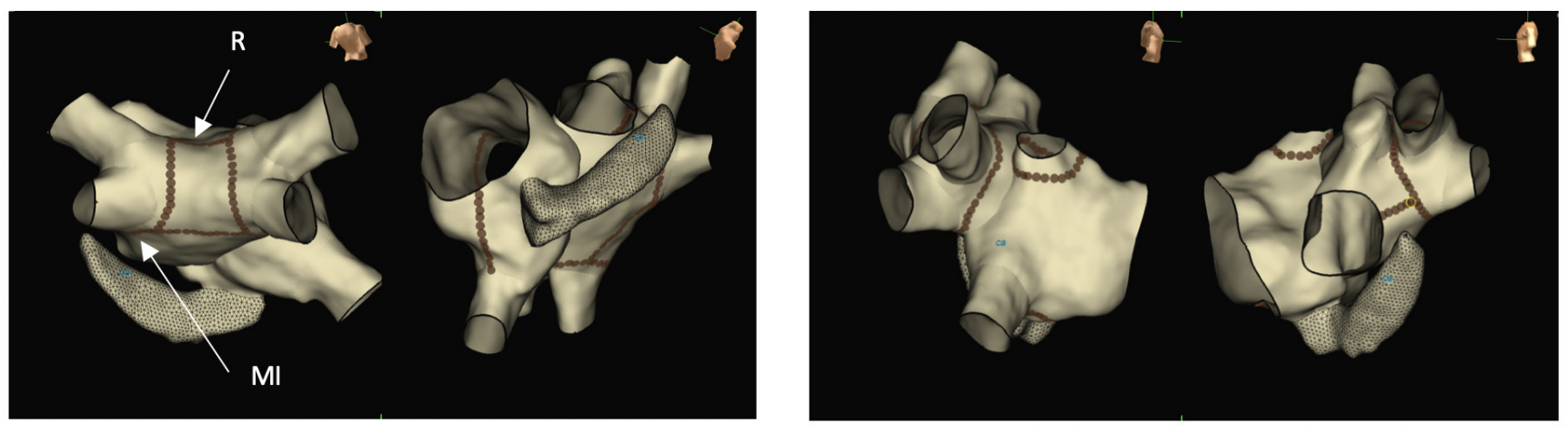

Figure 3 Linear ablation at mitral isthmus and roof line. MI, mitral isthmus; R, roof.

similar results when the recurrence rates of AF or AT at 18 months follow-up were compared between stand-alone PVI and PVI with additional ablation techniques. ${ }^{34}$

\section{Linear lesions}

The roof and mitral isthmus of the LA are the most commonly targeted sites for substrate modification using linear ablation (figure 3). However, it is difficult to achieve a durable and complete bidirectional conduction block across these lines. In particular, mitral isthmus ablation is difficult and may warrant entry into the coronary sinus to achieve complete block. This has been correlated with a high incidence of mitral isthmus reconduction, ${ }^{40}$ which is significantly associated with the development of mitral isthmus-dependent flutter. In a retrospective cohort of 50 patients who had perimitral flutters during the index procedure of follow-up, ${ }^{41}$ prior mitral isthmus ablation was associated with a higher risk of developing perimitral flutter despite achieving bidirectional block acutely.

Previous studies have demonstrated that up to $90 \%$ of ATs after AF ablation are re-entrant ${ }^{42}$ and consequently, repeated procedures are often required to increase the success rate above $50 \%$. However, even after multiple procedures, the incidence of atypical flutter or AT has been reported as high as $30 \% .{ }^{43}$ Therefore, while linear lesions may contribute to eliminating AF at initial ablation, incomplete block lines may promote proarrhythmic $\mathrm{AT}$ and re-entry.

\section{Rotor ablation}

$\mathrm{AF}$ has been postulated to be sustained by electrical rotors and focal impulses and ablation of such sources has been associated with improved procedural outcomes compared with conventional ablation alone. ${ }^{44}$ This is of particular interest in patients with long-standing persistent $\mathrm{AF}$, where the LAA may be a common source of AF rotors or focal drivers. Rotors are enabled by the ionic and conduction defects at the cellular level and intermittent areas of fibrosis lead to the stabilisation and propagation of rotors. ${ }^{45}$ Certain techniques, such as optical mapping, assist in uncovering the location of rotors and therefore, are amenable for ablation. Yet, there is no consensus on ablation of rotors in patients with AF due to inconsistencies in determining their contributory role in the maintenance of human AF. Most recently, focal impulse and rotor modulation (FIRM)-guided ablation demonstrated low long-term efficacy when performed in 43 patients as part of a single-centre observational study. ${ }^{46}$ FIRM-guided ablation led to redo PVI in $72 \%$ of patients, of which $21 \%$ were free from $\mathrm{AF}$ at $16 \pm 10.7$ months follow-up. Furthermore, the randomised BELIEF trial reported routine LAA isolation to be safe with no cases of embolic events postablation, although, an impaired contractile function of the LAA was identified in more than $50 \%$ of patients using transoesophageal echocardiography. ${ }^{47}$

The presence of rotors has been widely disputed given the available technologies used to map these small regions of rotational activity. Currently, the identification of rotors depends on utilising either basket catheters deployed within the atrium or multielectrode vests worn on the outside of the body to map AF. Both these mapping techniques lack detailed resolution and as result, identification of rotors can be challenging and heavily dependent on postprocessing of the raw data. The development of omnipolar technology may overcome these limitations by providing instantaneous visual depictions of local electrogram amplitude, activation direction and conduction velocity at any given point. Evidence supporting omnipolar mapping to conventional bipolar mapping has been previously described in vivo, ${ }^{48} 49$ but further investigation is warranted to validate this concept in human subjects.

\section{Scar homogenisation}

The pathophysiological importance of epicardial adipose tissue, atrial fibrosis and arrhythmogenic foci has been implicated in previous studies. The development and progression of atrial fibrosis are the primary modulator of structural remodelling in AF. Fibrosis promotes AF by interrupting fibre bundle continuity and causing disturbances in local conduction. ${ }^{50}$ Catheter ablation based on atrial scar sites as arrhythmic substrate has, therefore, emerged as a recent treatment approach for persistent AF. It can be detected from cardiac MRI with delayed enhancement or 3D mapping with bipolar low-voltage 
electrogram. Previous studies have demonstrated that atrial fibrosis, estimated by delayed-enhancement MRI, is independently associated with the likelihood of $\mathrm{AF}$ recurrence ${ }^{50} 51$ With evolving data on the association between atrial fibrosis and AF progression, ablation strategies have been developed to eliminate low-voltage zones that may indicate scar, otherwise known as scar homogenisation. Improved AF outcomes may be achievable through patient-specific substrate modification based on targeted ablation of atrial fibrosis. Nonetheless, voltage map-guided ablation is yet to gain widespread adoption into routine practice and further investigation is required to establish its long-term safety.

\section{CONCLUSION}

Despite the advancement of ablation strategies over the past two decades, long-term success rates and singleprocedural efficacy remain suboptimal for many patients. These outcomes are particularly evident in patients with persistent or long-standing $\mathrm{AF}$, where the results are clearly inferior to those for paroxysmal AF. Furthermore, the overall stroke and mortality benefits conferred on patients undergoing catheter ablation remain inconclusive. Ongoing clinical trials have, therefore, been designed to better address this. The Early treatment of Atrial Fibrillation for Stroke prevention Trial (EASTAFNET 4) has enrolled over 2500 and will evaluate whether an early, comprehensive, rhythm control strategy using anti-arrhythmic drugs (AADs) or catheter ablation prevents adverse cardiovascular outcomes compared with standard care. Other recently conducted trials such as CASTLE-AF assess these outcomes in specific patient populations, including those with heart failure and left ventricular ejection fraction below $35 \%$. With the development of ablation techniques, our understanding of $\mathrm{AF}$ mechanisms as well as the underlying factors influencing the incidence of perioperative complications, and overall success rates, are expected to improve. Thus, ongoing research may offer new treatment avenues for AF, while identifying novel therapeutic mechanisms in discrete populations of difficult-to-treat arrhythmia.

\section{Twitter Kathryn Lauren Hong @kathrynhong}

Contributors All authors contributed equally to this work.

Funding The authors have not declared a specific grant for this research from any funding agency in the public, commercial or not-for-profit sectors.

Competing interests None declared.

Patient consent for publication Not required.

Provenance and peer review Not commissioned; externally peer reviewed.

Data availability statement No data are available. Review article.

Open access This is an open access article distributed in accordance with the Creative Commons Attribution Non Commercial (CC BY-NC 4.0) license, which permits others to distribute, remix, adapt, build upon this work non-commercially, and license their derivative works on different terms, provided the original work is properly cited, appropriate credit is given, any changes made indicated, and the use is non-commercial. See: http://creativecommons.org/licenses/by-nc/4.0/.

ORCID iD
Kathryn Lauren Hong http://orcid.org/0000-0002-5542-7445

\section{REFERENCES}

1 Kirchhof P, Benussi S, Kotecha D, et al. Esc guidelines for the management of atrial fibrillation developed in collaboration with EACTS. Europace 2016;2016:1609-78.

2 Mujović N, Marinković M, Lenarczyk R, et al. Catheter ablation of atrial fibrillation: an overview for clinicians. Adv Ther 2017;34:1897-917.

3 Kirchhof P, Benussi S, Kotecha D, et al. Esc guidelines for the management of atrial fibrillation developed in collaboration with EACTS: the task force for the management of atrial fibrillation of the European Society of cardiology (ESC) developed with the special contribution of the European heart rhythm association (EHRA) of the $\mathrm{Sc}$ endorsed by the European stroke organisation (ESO). Europace 2016;2016:1609-78.

4 Ganesan AN, Shipp NJ, Brooks AG, et al. Long-Term outcomes of catheter ablation of atrial fibrillation: a systematic review and metaanalysis. J Am Heart Assoc 2013;2:e004549.

5 Calkins H, Hindricks G, Cappato R, et al. 2017 HRS/EHRA/ECAS/ APHRS/SOLAECE expert consensus statement on catheter and surgical ablation of atrial fibrillation: Executive summary. J Interv Card Electrophysiol 2017;50:1-55.

6 Jalife J, Berenfeld O, Mansour M. Mother rotors and fibrillatory conduction: a mechanism of atrial fibrillation. Cardiovasc Res 2002;54:204-16.

7 Haïssaguerre M, Hocini M, Sanders P, et al. Localized sources maintaining atrial fibrillation organized by prior ablation. Circulation 2006;113:616-25.

8 Brooks AG, Stiles MK, Laborderie J, et al. Outcomes of longstanding persistent atrial fibrillation ablation: a systematic review. Heart Rhythm 2010;7:835-46.

9 Kuck K-H, Brugada J, Fürnkranz A, et al. Cryoballoon or radiofrequency ablation for paroxysmal atrial fibrillation. $N$ Engl J Med 2016;374:2235-45.

10 Aryana A, Singh SM, Kowalski M, et al. Acute and long-term outcomes of catheter ablation of atrial fibrillation using the secondgeneration Cryoballoon versus Open-Irrigated radiofrequency: a multicenter experience. J Cardiovasc Electrophysiol 2015;26:832-9.

11 Andrade JG, Khairy P, Dubuc M. Catheter cryoablation: biology and clinical uses. Circ Arrhythm Electrophysiol 2013;6:218-27.

12 Barkagan M, Contreras-Valdes FM, Leshem E, et al. High-Power and short-duration ablation for pulmonary vein isolation: safety, efficacy, and long-term durability. J Cardiovasc Electrophysiol 2018;29:1287-96.

13 Bourier F, Duchateau J, Vlachos K, et al. High-Power short-duration versus standard radiofrequency ablation: insights on lesion metrics. J Cardiovasc Electrophysiol 2018;29:1570-5.

14 Kuck K-H, Reddy VY, Schmidt B, et al. A novel radiofrequency ablation catheter using contact force sensing: Toccata study. Heart Rhythm 2012;9:18-23.

15 Reddy VY, Shah D, Kautzner J, et al. The relationship between contact force and clinical outcome during radiofrequency catheter ablation of atrial fibrillation in the TOCCATA study. Heart Rhythm 2012;9:1789-95.

16 Kautzner J, Neuzil P, Lambert H, et al. EFFICAS II: optimization of catheter contact force improves outcome of pulmonary vein isolation for paroxysmal atrial fibrillation. Europace 2015;17:1229-35.

17 Natale A, Reddy VY, Monir G, et al. Paroxysmal AF catheter ablation with a contact force sensing catheter: results of the prospective, multicenter SMART-AF trial. J Am Coll Cardiol 2014;64:647-56.

18 De Groot JR, Berger WR, Krul SPJ, et al. Electrophysiological evaluation of thoracoscopic pulmonary vein isolation. $J$ Atr Fibrillation 2013;6:899.

19 Hussein AA, Barakat AF, Saliba WI, et al. Persistent atrial fibrillation ablation with or without contact force sensing. J Cardiovasc Electrophysiol 2017;28:483-8.

20 Lee G, Hunter RJ, Lovell MJ, et al. Use of a contact force-sensing ablation catheter with advanced catheter location significantly reduces fluoroscopy time and radiation dose in catheter ablation of atrial fibrillation. Europace 2016;18:211-8.

21 Ullah W, McLean A, et al, UK Multicentre Trials Group. Randomized trial comparing pulmonary vein isolation using the SmartTouch catheter.

22 Pedrote A, Arana-Rueda E, Arce-León A, et al. Impact of contact force monitoring in acute pulmonary vein isolation using an anatomic approach. A randomized study. Pacing Clin Electrophysiol 2016;39:361-9.

23 Reddy VY, Sediva L, Petru J, et al. Durability of pulmonary vein isolation with Cryoballoon ablation: results from the sustained PV 
isolation with Arctic front advance (SUPIR) study. J Cardiovasc Electrophysiol 2015;26:493-500.

24 Lin W-S, Tai C-T, Hsieh M-H, et al. Catheter ablation of paroxysmal atrial fibrillation initiated by non-pulmonary vein ectopy. Circulation 2003;107:3176-83.

25 Tamborero D, Mont L, Berruezo A, et al. Circumferential pulmonary vein ablation: does use of a circular mapping catheter improve results? A prospective randomized study. Heart Rhythm 2010;7:612-8.

26 Hong KL, Redfearn D, Chacko S, et al. High-Resolution mapping of the atria using the HD grid catheter. HeartRhythm Case Rep 2019:5:351-3 https://doi.org/

27 Bhardwaj R, Reddy VY. Visually-guided Laser Balloon Ablation of Atrial Fibrillation: A "Real World" Experience. Rev Esp Cardiol 2016;69:474-6.

28 Mujovic NM, Marinkovic MM, Potpara TS, et al. Catheter ablation of lone atrial fibrillation. Curr Pharm Des 2015;21:591-612

29 Wang X-H, Liu X, Sun Y-M, et al. Pulmonary vein isolation combined with superior vena cava isolation for atrial fibrillation ablation: a prospective randomized study. Europace 2008;10:600-5.

30 de Groot JR, Berger WR, Krul SPJ, et al. Electrophysiological evaluation of thoracoscopic pulmonary vein isolation. $J$ Atr Fibrillation 2013;6:899.

31 Okamatsu H, Okumura K. Strategy and Outcome of Catheter Ablation for Persistent Atrial Fibrillation - Impact of Progress in the Mapping and Ablation Technologies. Circ J 2017;82:2-9.

32 Haïssaguerre M, Sanders P, Hocini M, et al. Catheter ablation of long-lasting persistent atrial fibrillation: critical structures for termination. J Cardiovasc Electrophysiol 2005;16:1125-37.

33 Verma A, Jiang C-yang, Betts TR, et al. Approaches to catheter ablation for persistent atrial fibrillation. $N$ Engl $\mathrm{J} \mathrm{Med}$ 2015;372:1812-22.

34 Viles-Gonzalez JF, Gomes JA, Miller MA, et al. Areas with complex fractionated atrial electrograms recorded after pulmonary vein isolation represent normal voltage and conduction velocity in sinus rhythm. Europace 2013;15:339-46.

35 Lo L-W, Lin Y-J, Chang S-L, et al. Beyond pulmonary vein isolation: the role of additional sites in catheter ablation of atrial fibrillation. Curr Cardiol Rep 2017;19:86.

36 RASTA

37 Vogler J, Willems S, Sultan A, et al. Pulmonary vein isolation versus defragmentation: the CHASE-AF clinical trial. J Am Coll Cardiol 2015;66:2743-52 https://doi.org/

38 Fink T, Schlüter M, Heeger $\mathrm{CH}$, et al. Stand-Alone pulmonary vein isolation versus pulmonary vein isolation with additional substrate modification as index ablation procedures in patients with persistent and long-standing persistent atrial fibrillation: the randomized Alster-LostAF trial (ablation at St. Georg Hospital for long-standing persistent atrial fibrillation). Circ Arrhythm Electrophysiol 2017:10 https://doi.org/

39 Sawhney N, Anousheh R, Chen W, et al. Circumferential pulmonary vein ablation with additional linear ablation results in an increased incidence of left atrial flutter compared with segmental pulmonary vein isolation as an initial approach to ablation of paroxysmal atrial fibrillation. Circulation 2010;3:2-248.

40 Matsuo S, Wright M, Knecht S, et al. Peri-mitral atrial flutter in patients with atrial fibrillation ablation. Heart Rhythm 2010;7:2-8.

41 Chae S, Oral H, Good E, et al. Atrial tachycardia after circumferential pulmonary vein ablation of atrial fibrillation: mechanistic insights, results of catheter ablation, and risk factors for recurrence. J Am Coll Cardiol 2007;50:1781-7.

42 Lo L-W, Lin Y-J, Chang S-L, et al. Predictors and characteristics of multiple (more than 2) catheter ablation procedures for atrial fibrillation. J Cardiovasc Electrophysiol 2015;26:1048-56.

43 Narayan SM, Krummen DE, Shivkumar K, et al. Treatment of atrial fibrillation by the ablation of localized sources: confirm (conventional ablation for atrial fibrillation with or without focal impulse and rotor modulation) trial. J Am Coll Cardiol 2012;60:628-36.

44 Krummen DE, Swarup V, Narayan SM. The role of rotors in atrial fibrillation. J Thorac Dis 2015;7:142-51.

45 Steinberg JS, Shah Y, Bhatt A, et al. Focal impulse and rotor modulation: acute procedural observations and extended clinical follow-up. Heart Rhythm 2017;14:192-7.

46 Di Biase L, Burkhardt JD, Mohanty P, et al. Left Atrial Appendage Isolation in Patients With Longstanding Persistent AF Undergoing Catheter Ablation: BELIEF Trial. J Am Coll Cardiol 2016;68:1929-40.

47 Deno DC, Balachandran R, Morgan D, et al. Orientation-Independent Catheter-Based characterization of myocardial activation. IEEE Trans Biomed Eng 2017:64:1067-77.

48 Massé S, Magtibay K, Jackson N, et al. Resolving myocardial activation with novel omnipolar electrograms. Circ Arrhythm Electrophysiol 2016;9:e004107.

49 Hsieh M-H, Tai C-T, Lee S-H, et al. The different mechanisms between late and very late recurrences of atrial fibrillation in patients undergoing a repeated catheter ablation. $J$ Cardiovasc Electrophysiol 2006;17:231-5.

50 Marrouche NF, Wilber D, Hindricks G, et al. Association of atrial tissue fibrosis identified by delayed enhancement MRI and atrial fibrillation catheter ablation: the DECAAF study. JAMA 2014;311:498-506. 